\section{Histochemical Adaptation of Aryl lodosoacetates}

IT has been possible to obtain cleavaige of the 1,2 glycol linkage in tissue sections with a solution of phenyl iodosoacetate in glacial acetic acid ${ }^{1}$, thus duplicating histochemically the chemical studies of Criegee and Beucker ${ }^{2}$. However, to establish definitely the reliability of aryl iodosoacetates as histochemical reagents, it was felt that several other com. pounds of this type should be examined in a similar manner.

Four aryl iodosoacetates were prepared by Willgerodt's general methods ${ }^{3}$, namely, $p$-nitrophenyl iodosoacetate, $p$-tolyl iodosoacetate, $m$-tolyl iodosoacetate, 2,4 xylyl iodosoacetate. These compounds were then tested histochemically for 1,2 glycol cleavage using formalin-fixed sections (paraffin technique) of rat and rabbit intestine and kidney. Concentrations of these compounds in glacial acetic acid were used ranging from $0.001 M$ to saturation, with oxidation periods of $15 \mathrm{~min} .-3 \mathrm{hr}$. All reactions were performed at room temperature $\left(27^{\circ} \mathrm{C}\right.$.), and the aldehyde radicals resulting from the cleavage were stained with leucofuchsin. The $0.02 M$ solutions suggested by Pausacker for chemical cleavage of isohydrobenzoin and used in the phenyl iodosoacetate experiments appeared to be the concentration of choice, while the period of optimal oxidation was between 1 and $2 \mathrm{hr}$. The histological picture was similar to the one obtained with phenyl iodosoacetate and resembled that of lead tetraacetate ${ }^{5}$ although weaker and less precise.

These experiments would appear to indicate that aryl iodosoacetates as a group are adaptable to histochemical procedures, and although their use is still academic, they may be of value to histochemistry in due course.

These experiments were made possible through a research grant by the Biological Stain Commission.

\section{JohN F. LнотkA}

Department of Anatomy,

University of Oklahoma School of Medicine, Oklahoma City, Oklahoma. July 1.

1 Lhotka, J. F., Stain Tech. (in the press).

Criegee, R., and Beucker, H., Annaten, 541, 218 (1939).

- Willgerodt, C., J. prak. Chem., 33, 156 (1886); 59, 198 (1899).

- Pausacker, K. H., J. Chem. Soc., 107 (1953).

'Lhotka, J. F., Stain Tech., 27, 213 (1952).

\section{Chromatography of Tissue Histamine}

Extracts of tissues exceptionally rich in histamine, such as ox pleura, ox liver capsule, and mast cell tumours from $\operatorname{dogs}^{1}$, were prepared for ascending chromatographic estimation of histamine by simple extraction or by grinding the tissues with 10 per cent aqueous trichloracetic acid and centrifuging. Using as solvent mixture $n$-butanol : acetic acid : water (4:1:5), we observed that after spraying with the Pauly or the paranitraniline diazo-reagent two sharply defined spots appeared on the chromatogram of $R_{F}=0.11$ and 0.65 (Fig. 1,A). Both spots contained material which when eluted with water or $0.01 \mathrm{~N}$ hydrochloric acid showed histamine activity on the isolated guinea pig ileum and on the blood pressure of the atropinized cat. Prior removal of the excess trichloracetic acid from the solution by

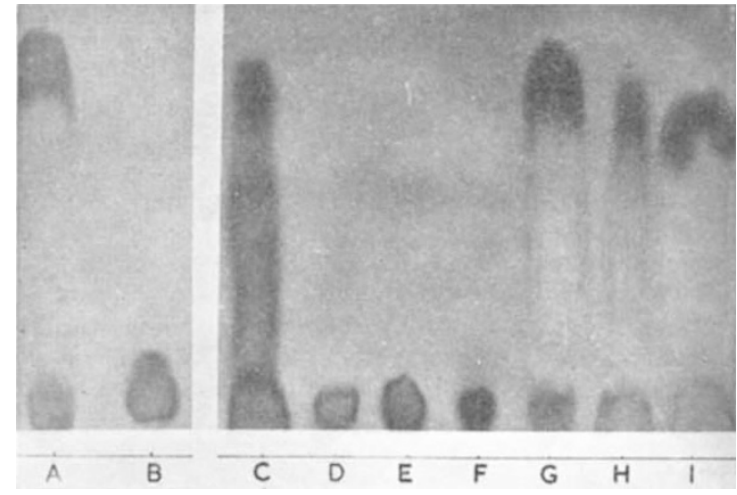

Fig. 1. Chromatograms developed with Pauly diazo-reagent for histamine. (A) Extract of dog mastocytoma in 10 per cent (C) solution of histamine $(100 \mu \mathrm{gm}$.) in 10 per cent trichloracetic acid ; (D) solution of histamine (100 $\mu \mathrm{gm}$.) in $N / 3$ hydrochloric acid; $(E)$ solution $C$ after ether extraction: $\left(F^{\prime}\right)$ solution of histamine $(30 \mu \mathrm{gm}$.) in 10 per cent trichloracetic acid $(G)$ solution $F$ with $300 \mu \mathrm{gm}$. arginine added $;(H)$ solution $G$ after ether extraction; $(I)$ solution $F^{\prime}$ with $100 \mu \mathrm{gm}$. each of arginine, lysine and ornithine added

ether extraction merely reduced the intensity of the fast-running spot. When an eluate of this fastrunning spot $\left(R_{F}=0.65\right)$ was re-run in the same solvent as before, the histamine now remained entirely in its lower location $\left(R_{F}=0 \cdot 1 \mathrm{I}\right)$.

At least three factors appear to be involved in the migration of histamine to the fast-running spot, namely : (1) the concentration of trichloracetic acid ; (2) the concentration of histamine; (3) the presence of a basic amino-acid. First, pure histamine acid phosphate in water forms a compact spot at $R_{F}=0 \cdot 11$ (Fig. 1,B), but with increasing concentration of trichloracetic acid a 'trail' of histamine moves up the paper as far as the $R_{F}=0.65$ area (Fig. $1, C$ ). This is not a mere $p H$ effect, for histamine in $0.33 \mathrm{~N}$ hydrochloric acid (Fig. $1, D$ ) or in 10 per cent aqueous acetic acid does not migrate in this way. Trifluoracetic acid resembles trichloracetic acid in its mobilizing effect on histamine, recalling the similar effect of these acids on the chromatographic behaviour of adrenaline ${ }^{2}$. Removal of the excess acid from these solutions by ether extraction concentrates the histamine into a single compact spot at $R_{F}=0 \cdot 11$ (Fig. 1,E). Secondly, when the strength of the trichloracetic acid is kept constant, any increase in concentration of histamine in the mixture is followed by increased upward trailing from the slow-running spot (Fig. 1, ef. $C$ and $F$ ). Thirdly, for complete separation of the two spots, the presence of at least one basic amino-acid is necessary. When an artificial mixture is made up containing pure histamine, trichloracetic acid and arginine, lysine or ornithine, the chromatographic pattern now exactly resembles that seen with tissue extracts, the histamine being divided between the two spots, $R_{F}=0.11$ and $R_{F}=0.65$ (Fig. 1,G); ether extraction of this mixture simply reduces the intensity of the fast-running spot (Fig. $1, H)$. When all three amino-acids are present in the mixture, the chromatogram shows inversion (Fig. $1, I)$.

It is thus possible that the remarkable ability of trichloracetic acid to extract histamine from tissues is related not only to its coagulative effect on protein but also to its participation in a loose complex containing histamine (probably as the trichloracetate) and basic amino-acids. Detailed analysis of the 